\title{
Reuna
}

\section{DESENVOLVIMENTO SUSTENTÁVEL E COMPETITIVIDADE DAS NAÇÕES: UMA ANÁLISE MULTIVARIADA}

\author{
SUSTAINABLE DEVELOPMENT AND NATIONAL COMPETITIVENESS: A \\ MULTIVARIATE ANALYSIS
}

http://dx.doi.org/10.21714/2179-8834/2017v22n1p41-62

\author{
Magno Luís Bezerra Lima \\ Universidade Federal do Ceará, Brasil. \\ Endereço: Avenida da Universidade, 2431 Benfica, CEP 60020180 - Fortaleza, CE - Brasil \\ Fone: $+55(085) 33667800$ \\ Email: magnoluis.eq@gmail.com- Lattes: http://lattes.cnpq.br/2031899511755660 \\ Julynara Alves Frota \\ Universidade Federal do Ceará, Brasil. \\ Endereço: Avenida da Universidade, 2431 Benfica, CEP 60020180 - Fortaleza, CE - Brasil \\ Fone: (085) 33667800 \\ Email: julynarafrota@gmail.com- Lattes: http://lattes.cnpq.br/9493308138814957 \\ Francisco Sávio Maurício Araújo \\ Universidade Federal do Ceará, Brasil. \\ Endereço: Avenida da Universidade, 2431 Benfica, CEP 60020180 - Fortaleza, CE - Brasil \\ Fone: $+55(085) 33667800$ \\ Email: franciscosavio@outlook.com- Lattes: http://lattes.cnpq.br/7896432127948510 \\ Raimundo Eduardo Silveira Fontenele \\ Universidade Federal do Ceará, Brasil. \\ Endereço: Avenida da Universidade, 2431 Benfica, CEP 60020180 - Fortaleza, CE - Brasil \\ Fone: $+55(085) 33667800$ \\ Email: prof.eduardo.fontenele@gmail.com- Lattes: http://lattes.cnpq.br/5547677980453020
}

Submissão: 15 Fev. 2017 Publicação: 31 Mar. 2017. Sistema de avaliação: Double blind review. Centro Universitário UNA, Belo Horizonte - MG, Brasil. Editor geral Prof. Dr. Mário Teixeira Reis Neto

Este artigo encontra-se disponível no endereço eletrônico:

http://revistas.una.br/index.php/reuna/article/view/877

http://dx.doi.org/10.21714/2179-8834/2017v22n1p41-62

\section{Resumo}

Nas últimas décadas, o conceito de Desenvolvimento Sustentável faz parte das estratégias das nações, como uma forma de promover a competitividade, bem como de mantê-la nas ações em longo prazo. De acordo com a definição da Comissão Brundtland, desenvolvimento sustentável é quando as necessidades da geração atual são satisfeitas sem comprometerem a capacidade das gerações futuras de atenderem às suas próprias necessidades. A meta principal da competitividade nacional é melhorar o padrão e a qualidade de vida da população, por meio da ampliação da capacidade empresarial nos mercados internacionais. Neste contexto, 
o presente trabalho busca investigar a influência do desenvolvimento sustentável entre os países. Para isso, avaliou-se quantitativamente a relação entre indicadores associados à competitividade e desenvolvimento sustentável, com base em vários indicadores, tais como: Índice de Performance Ambiental (EPI Environmental Performance Index) e Objetivos do Desenvolvimento Sustentável (SDG Sustainable Development Goals), os quais foram utilizados nesta análise. Para avaliar a competitividade, utilizou-se o Índice de Competitividade Global (GCI). Verificou-se que o SDG teve maior impacto sobre o $\mathrm{GCl}$, enquanto o PIB mostrou a correlação mais significativa com o $\mathrm{GCl}$. Os resultados também mostraram que há um trade off entre a competitividade e fatores sociais / ambientais dentro de cada grupo obtidos pela análise de cluster.

Palavras-chave: sustentabilidade, Gestão ambiental, Competitividade.

\begin{abstract}
The concept of Sustainable Development has proven to be decisive in order to improve and maintain the competitiveness of a country in a long term. According to the Brutland report, sustainable development is development that meets the needs of the present without compromising the ability of future generations to meet their own need. On the other hand, the competitiveness of a nation aims to improve the standard of living of the population by enhancing its entrepreneurship in the international market. In this context, the main objective of this paper is to investigate the effect of sustainable development throughout the nations in the globe. Thus, the relation among indexes related to competitiveness and sustainability, such as EPI (Environmental Performance Index) and SDG (Sustainable Development Goals), was quantitatively evaluated. The $\mathrm{GCl}$ (Global Competitiveness Index) was utilized in order to assess a country's level of competiveness. The SDG was found to have the greatest impact on the $\mathrm{GCl}$, whereas the GDP showed the most significant correlation with the $\mathrm{GCl}$. The results also showed that there is a tradeoff between competitiveness and social/environmental factors within each group obtained by the cluster analysis.
\end{abstract}

Keywords: Sustainability, Environmental Management, Competitiveness.

\title{
1. Introdução
}

Durante o crescimento industrial, no final do século XIX, acreditava-se que o crescimento econômico não tinha limites e que o desenvolvimento significava dominar a natureza e os homens. Porém, neste mesmo século, houve uma grande mudança na mentalidade dos homens no que diz respeito à relação sociedadenatureza (SCHLINDWEIN; JUCHEM; RAFUL, 2009).

Após a Segunda Guerra Mundial, surge então uma discussão quanto à consciência ambiental, conhecida como a "revolução ambiental". Assim, a humanidade percebe que os recursos naturais não são ilimitados e que o uso descontrolado destes pode acarretar graves consequências (SCHLINDWEIN; JUCHEM; RAFUL, 2009). 
Anos depois, na Conferência das Nações Unidas em 1972, que ficou conhecida como a Conferência de Estocolmo, foi apontada a devastação da natureza que ocorria naquele momento e determinou-se que o crescimento humano precisaria ser repensado imediatamente (PEDRINI, 1998). Foi então, sob o impacto crescente das "externalidades" econômicas, tais como o acidente nuclear de Chernobyl, em 1986, que contaminou boa parte da Europa, quando se estruturou uma das mais importantes iniciativas para a promoção de parâmetros éticos e ambientais para a produção humana na Terra (MARCONDES; BACARJI, 2010).

Em abril de 1987, o documento chamado de Nosso futuro Comum, também conhecido como Relatório Brundtland, elaborado pela Comissão Mundial sobre o Meio Ambiente e Desenvolvimento da ONU, descreveu os riscos provenientes das limitações da biosfera em absorver os efeitos do crescimento da sociedade humana. $\mathrm{Na}$ época, embora os efeitos desastrosos do aquecimento global ainda não estivessem tão evidentes como nos dias atuais, o relatório apresentado já destacava a necessidade de uma maior atenção aos recursos naturais durante o crescimento econômico. O objetivo deste documento era a formação de um acordo mundial que planejasse um futuro em que não ocorresse exaustão dos recursos ambientais e em que as desigualdades entre pobres e ricos fossem reduzidas (DOCUMENTO DAS NAÇÕES UNIDAS, 1987).

Nos últimos anos, o conceito de Desenvolvimento Sustentável vem ganhando importância nas estratégias das nações como uma forma de promover a competitividade, e também de mantê-la em longo prazo. Essa disputa deve ser saudável ao mercado, sem que haja danos à disponibilidade de recursos para gerações futuras, das quais as empresas ou nações dependerão para se manterem competitivas.

Neste contexto, o problema desta pesquisa é investigar: Como a sustentabilidade impacta no desenvolvimento entre os países? Qual a relação entre indicadores associados à competitividade e desenvolvimento sustentável?

O presente artigo está estruturado em quatro seções, além desta introdução. Na segunda seção, como referencial teórico, faz-se uma breve discussão sobre os conceitos de desenvolvimento sustentável, competitividade, sustentabilidade e seus indicadores; na seção seguinte, os procedimentos metodológicos são descritos; a seguir, os resultados são analisados e discutidos; por fim, são apresentadas as principais conclusões.

\section{Desenvolvimento sustentável, Competitividade, Sustentabilidade}

Para o desenvolvimento econômico de uma nação, sabe-se que a questão ambiental constitui um dos fatores indispensáveis. Sendo assim, no cenário mundial, tem-se discutido muito sobre estratégias de prosperidade e desenvolvimento, tendo como foco a competitividade e a sustentabilidade.

Souza (2002) define o desenvolvimento econômico a partir do "crescimento econômico contínuo, em ritmo superior ao crescimento demográfico, em que haja mudanças de estruturas e melhoria de indicadores econômicos, sociais e ambientais". Segundo ele, as consequências do desenvolvimento com a 
preservação do meio ambiente em longo prazo são: o fortalecimento da economia nacional, o crescimento da economia de mercado e a elevação geral da produtividade e do nível de bem-estar geral da população.

Neste contexto, os tópicos abaixo visam apresentar uma discussão mais aprofundada dos conceitos de desenvolvimento sustentável, competitividade, sustentabilidade e os seus indicadores.

\subsection{Desenvolvimento sustentável}

De acordo com a definição da Comissão Brundtland, desenvolvimento sustentável é quando as necessidades da geração atual são satisfeitas sem comprometerem a capacidade das gerações futuras de atenderem às suas próprias necessidades.

A definição acima aponta dois conceitos-importantes, o conceito de "necessidades", principalmente as necessidades básicas dos pobres do mundo, que devem ser prioridade absoluta; e a ideia de barreiras impostas pelo Estado, com uso da tecnologia e da organização social sobre a capacidade do ambiente em atender às necessidades atuais e futuras (DOCUMENTO DAS NAÇÕES UNIDAS, 1987).

Mesmo os conceitos de desenvolvimento sustentável e sustentabilidade apresentando semelhanças, torna-se comum relacionar desenvolvimento sustentável a políticas públicas e sustentabilidade às demais ações.

Contudo, o conceito de desenvolvimento sustentável é como uma ferramenta para o crescimento que une o desenvolvimento econômico, a proteção do ambiente e a justiça social, sendo seus valores reconhecidos pelos governos democráticos e os movimentos políticos do mundo todo.

\subsection{Competitividade}

Segundo a Teoria Neoclássica descrita por Porter (1986), a competitividade nada mais é que uma questão de preços, custos, taxas de câmbio, dentre outros pontos, como estando aparentemente superados. Nesse mesmo momento, Porter confirmou essa Teoria, levando muitos países a manipularem tais fatores com a intenção de alcançarem vantagens na competição mundial.

Numa empresa, a competitividade é definida como a habilidade de produzir bens e serviços certos, de boa qualidade, pelo preço justo, no momento apropriado, satisfazendo assim as expectativas dos clientes de forma mais competente e mais eficaz que nas empresas concorrentes (EDMONDS, 2000). Já a competitividade nacional é determinada pela capacidade de um país em desenvolver, produzir, distribuir produtos e serviços no comércio internacional ao perceber um aumento nos retornos sobre seus recursos (ARSLAN; TATHDIL, 2012).

Portanto, o conceito de competitividade e a busca da vantagem competitiva, seja dentro da empresa ou por uma nação, fundamenta-se, principalmente, nos aumentos de produtividade e nos valores agregados dispostos no processo produtivo e social. Então, verificar os fatores que determinam esse aumento de 
produtividade e o seu crescimento real num determinado espaço de tempo constituise como um dos mais importantes objetivos da análise do desenvolvimento econômico.

Baseada nos mais recentes estudos neste campo de pesquisa, a competitividade (em nível de nação) pode ser definida como o conjunto de instituições, políticas e fatores que determinam o nível de produtividade de um país (SALA-I-MARTIN et al., 2013). Seu objetivo maior é ser a base de uma qualidade de vida elevada, fazendo com que esse conceito vá muito além dos padrões de vida materiais. A meta principal da competitividade nacional é melhorar o padrão e a qualidade de vida da população, através da ampliação da capacidade empresarial dos municípios ao comércio nos mercados internacionais.

Competitividade induz os países à criação de um bom desempenho financeiro nacional, bem-estar para os cidadãos e prosperidade sustentável para a economia. Então, para as economias mundiais, tornou-se um objetivo alcançar um alto padrão de competitividade. (CECILIA-NICOLETA; ALEXANDRU, 2015)

\subsection{Sustentabilidade}

Philippi (2001) afirma que sustentabilidade é a capacidade de se sustentar e de se manter, ou seja, uma atividade sustentável é aquela que pode ser mantida por um longo período de tempo, de forma a não ser afetada, apesar dos possíveis imprevistos que podem vir a acontecer durante este período. O conceito de sustentabilidade pode ser ainda ampliado, usando-se o conceito de uma sociedade sustentável, ou seja, que não coloca em risco os recursos naturais como o ar, a água, o solo e a vida vegetal e animal dos quais a vida (da sociedade) depende.

Vieira (2008) afirma que antigamente a sustentabilidade da sociedade humana era uma suposição, não um objetivo. As mudanças ambientais eram vagarosas e aceitavam retornos adaptativos ou modificações do ambiente sem muitas pressões quanto à sua velocidade. Hoje em dia, o crescimento rápido da população mundial e o alto consumo de bens e energia causam preocupação quanto à capacidade do meio e da sociedade de se manterem sustentáveis. O conceito de sustentabilidade inicialmente estava ligado à qualidade ambiental. Hoje, ele permeia diversos segmentos da sociedade.

Dentro desta perspectiva, fundamentada em um processo de mudanças, Sachs (1993) aponta que o conceito de desenvolvimento sustentável apresenta cinco dimensões principais: a) Sustentabilidade ecológica - referindo-se à parte física do processo de crescimento, a qual tem como objetivo a manutenção de estoques dos recursos naturais, incorporados às atividades produtivas; b) Sustentabilidade ambiental - referindo-se à preservação da capacidade de manutenção dos ecossistemas; c) Sustentabilidade social - referindo-se ao desenvolvimento, cujo objetivo é a melhoria da qualidade de vida da população. No caso de países com desigualdade social e baixo índice de inclusão social, significa a adoção de políticas distributivas e a universalização de atendimento a questões como saúde, educação, habitação e seguridade social; d) Sustentabilidade política - referindo-se ao processo de construção da cidadania para certificar a inclusão total dos indivíduos ao processo de desenvolvimento; e) Sustentabilidade econômica - referindo-se a um 
gerenciamento eficiente dos recursos em geral e caracterizando-se pela regularidade de fluxos do investimento público e privado.

\subsection{Indicadores de sustentabilidade}

Nesse contexto, os indicadores surgem como uma ferramenta para auxiliar nas avaliações sobre a sustentabilidade, cuja função é o estabelecimento de uma visão de conjunto que determina um processo de estimativa de resultados em relação aos objetivos de desenvolvimento sustentável estabelecidos, fornecendo às partes interessadas condições adequadas de acompanhamento e dando apoio ao processo decisório (MALHEIROS; PHILIPPI; COUTINHO, 2008).

Há vários indicadores que demonstram os diferentes aspectos do desenvolvimento sustentável, dentre eles destacam-se: Índice de Performance Ambiental (EPI Environmental Performance Index) e Objetivos do Desenvolvimento Sustentável (SDG - Sustainable Development Goals), os quais foram utilizados nesta análise.

Todavia, os indicadores não são e nem devem ser usados como soluções para todos os problemas que envolvem a sustentabilidade, seja na sua avaliação, seja na sua operacionalização (KEMERICH; RITTER; BORBA, 2014). Fernandes (2004) afirma que os indicadores, na verdade, exercem a sua função, ou seja, meramente indicam os meios para avaliação, para a discussão e para a percepção da sustentabilidade, permitindo a quem os utiliza realizar as demais etapas.

\subsection{1 Índice da Performance Ambiental}

O Índice da Performance Ambiental (Environmental Performance Index - EPI) é um índice apresentado pelas Universidades de Yale e Columbia, em que se faz a avaliação, em nível mundial, de como está o tema gestão ambiental em diversos países. Os índices compilam um ranking que aponta em quais países o meio ambiente está mais adequado ao desenvolvimento sustentável em 2 áreas: proteção da saúde humana e proteção dos ecossistemas (PNUD, 2000). A estrutura do EPI é apresentada na FIG.1 


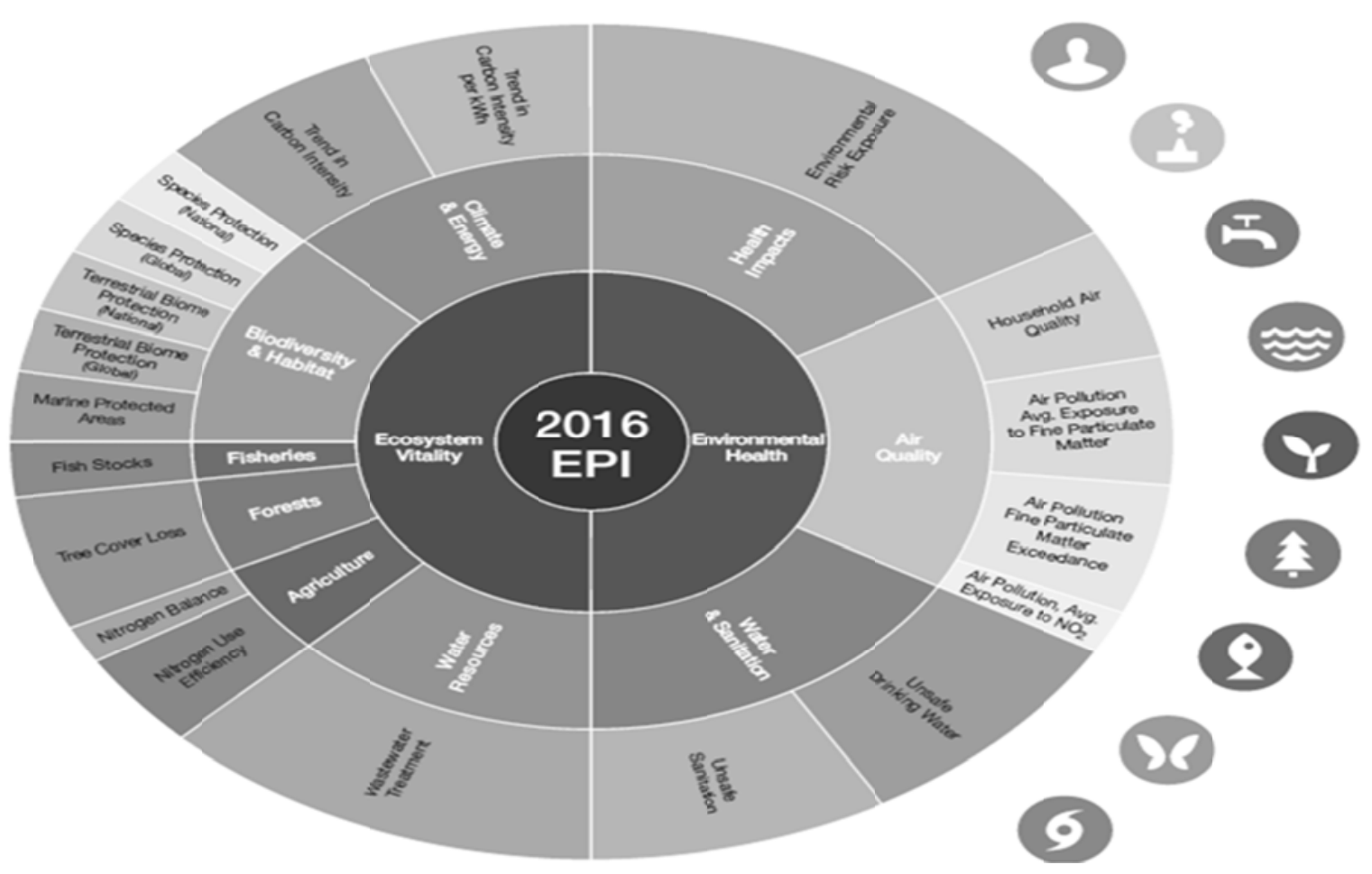

Figura 1- Estrutura do EPI.

Fonte: HSU et al (2016).

Nota: O acesso à eletricidade não está incluído na figura porque não é usado para calcular a pontuação de cada país.

O índice é produzido com o propósito de oferecer informações para as políticas públicas e contém 22 indicadores que analisam as mudanças ambientais, permitindo descrever os pontos fortes e fracos de vários países em 9 categorias temáticas que abrangem questões de política ambiental de alta prioridade, incluindo Agricultura, Qualidade do Ar, Biodiversidade e Habitat, Clima e Energia, Florestas, Pescas, Impactos sobre a Saúde, Recursos Hídricos e Água e Saneamento, em nível nacional. O índice encontra-se em sua quarta edição (HSU et al., 2016

\subsubsection{Objetivos do Desenvolvimento Sustentável}

Os 17 Objetivos do Desenvolvimento Sustentável (Sustainable Development Goals $S D G$ ) da Agenda 2030 - adotados por líderes mundiais, em setembro de 2015, numa histórica Cúpula da ONU - entraram oficialmente em vigor em 01 de Janeiro de 2016. Ao longo dos próximos 15 anos, com esses novos objetivos, que são universais, os países deverão mobilizar esforços para acabar com todas as formas de pobreza, lutar contra as desigualdades e combater as alterações climáticas (BM\&FBOVESPA, 2016).

Os 17 indicadores utilizam as informações mais atuais, abrangendo $80 \%$ de todos os países com a população acima de 1 milhão de pessoas. Estes são relacionados à pobreza, fome, saúde e bem-estar, educação de qualidade, igualdade de gênero, água limpa e saneamento, energia limpa, trabalho decente e crescimento econômico, inovação na indústria e infraestrutura, redução de desigualdades, 
cidades e comunidades sustentáveis, consumo e produção responsável, mudança climática, vida aquática, vida na Terra, paz, justiça e instituições fortalecidas, dentre outros (UNITED NATIONS, 2016), conforme mostra o quadro 1.

Quadro 1- Objetivos do Desenvolvimento Sustentável (SDG).

Objetivos do Desenvolvimento Sustentável (SDG)

1 Acabar com a pobreza em todas as suas formas, em todos os lugares.

2 Acabar com a fome, alcançar a segurança alimentar e melhoria da nutrição e promover a agricultura sustentável.

3 Assegurar uma vida saudável e promover o bem-estar para todos, em todas as idades.

4 Assegurar a educação inclusiva e equitativa e de qualidade, e promover oportunidades de aprendizagem ao longo da vida para todos.

$5 \quad$ Alcançar a igualdade de gênero e empoderar todas as mulheres e meninas.

6 Garantir disponibilidade e manejo sustentável da água e saneamento para todos.

7 Garantir acesso à energia barata, confiável, sustentável e renovável para todos.

8 Promover o crescimento econômico sustentado, inclusivo e sustentável, emprego pleno e produtivo, e trabalho decente para todos.

9 Construir infraestrutura resiliente, promover a industrialização inclusiva e sustentável, e fomentar a inovação.

10 Reduzir a desigualdade dentro dos países e entre eles.

11 Tornar as cidades e os assentamentos humanos inclusivos, seguros, resilientes e sustentáveis.

12 Assegurar padrões de produção e de consumo sustentáveis.

13 Tomar medidas urgentes para combater a mudança climática e seus impactos. ( $\left.{ }^{*}\right)$

14 Conservação e uso sustentável dos oceanos, dos mares e dos recursos marinhos para o desenvolvimento sustentável.

15 Proteger, recuperar e promover o uso sustentável dos ecossistemas terrestres, gerir de forma sustentável as florestas, combater a desertificação, deter e reverter a degradação da terra e deter a perda de biodiversidade.

16 Promover sociedades pacíficas e inclusivas para o desenvolvimento sustentável, proporcionar o acesso à justiça para todos e construir instituições eficazes, responsáveis e inclusivas em todos os níveis.

17 Fortalecer os meios de implementação e revitalizar a parceria global para desenvolvimento sustentável.

Fonte: UNITED NATIONS, 2016.

Trata-se de um ambicioso conjunto de objetivos nas três dimensões do desenvolvimento sustentável - desenvolvimento econômico, inclusão social e sustentabilidade ambiental, apoiada por um bom governo (UNITED NATIONS, 2016).

Com isso, o SDG se torna um instrumento prático para resolução de problemas, na medida em que mobiliza os governos, academias, sociedade civil e negócio; (b) fornece relatórios para rastrear o progresso e assegurar a responsabilização; (c) serve de ferramenta de gestão para as ações necessárias, a fim de atingir os objetivos de desenvolvimento sustentável para 2030 (UNITED NATIONS, 2016). 


\subsection{Indicadores de competitividade}

Desde os anos 90, instituições e agências internacionais, como o Banco Mundial, FMI, ONU, etc., têm publicado várias pesquisas e relatórios sobre os indicadores de desenvolvimento sustentável e competitividade nacional de diferentes países (ONU, 2001).

Nesta pesquisa foi utilizado o Índice de Competitividade Global (Global Competitiveness Index). O Fórum Econômico Mundial (WEF) define a competitividade nacional como um conjunto de organizações, políticas e fatores que determinam o nível de produtividade de um país (SCHWAB, 2009). O GCI foi criado pelo WEF com o objetivo de incorporar no conceito de competitividade diferentes níveis da abordagem para esta problemática - microeconômica, apresentados principalmente pela metodologia de análise da competitividade das empresas, e macroeconômica, geralmente utilizados para as comparações internacionais de países (KASIMOVSKAYA; DIDENKO, 2013)

O Índice de Competitividade Global ( $\mathrm{GCl}$ ) envolve 133 países de todas as regiões do mundo e comprova que competitividade nacional é um fenômeno complexo, mas que pode ser aperfeiçoado por meio de reformas em diversas áreas que influenciam a produtividade de um país em longo prazo (SCHWAB, 2009). O índice capta essa dimensão em aberto e apresenta uma média ponderada de diversos componentes, em que cada uma delas descreve um aspecto complexo do conceito que é chamado competitividade.

Empregando dados oriundos de organismos oficiais e de instituições supranacionais, entre os quais destacam-se o Fundo Monetário Internacional (FMI), o Banco Mundial (BM), a Organização das Nações Unidas (ONU) e a Organização para a Cooperação e Desenvolvimento Econômico (OCDE), o GCl tem, na sua gênese, mais de uma centena de indicadores (116) que possibilitam a contemplação de um número vasto de economias. Desta forma, o $\mathrm{GCl}$ atesta-se na condição de um índice abrangente em comparação com os seus homólogos (FÓRUM ECONÔMICO MUNDIAL, 2010).

Os 116 indicadores referidos concretizam-se em 12 pilares de competitividade, que representam áreas determinantes de competitividade e que, por seu turno, erguem três sub-índices, o dos requerimentos básicos, o dos fatores potenciadores de eficiência e dos fatores de inovação e sofisticação. $\mathrm{O} \mathrm{GCl}$ é a média dos três subíndices. (FÓRUM ECONÔMICO MUNDIAL, 2010).

Os 12 pilares são os seguintes: I - instituições; II - infraestruturas; III - ambiente macroeconômico; IV - saúde e educação primária; V - educação superior e formação; VI - eficiência dos mercados; VII - eficiência dos mercados de trabalho; VIII - eficiência dos mercados financeiros; IX - tecnologia disponível; X - dimensão dos mercados internos e externos; XI - sofisticação dos processos de produção; e XII - inovação (FÓRUM ECONÔMICO MUNDIAL, 2010).

\subsection{Curva Ambiental de Kuznets Tradicional}


A Curva Ambiental de Kuznets apresentada na FIG. 2 se apoia no conceito que surgiu no começo da década de 1990 para descrever os impactos que o desenvolvimento econômico exerce sobre os níveis de emissões de poluentes, uma vez que estes estão intimamente relacionados com a produção de um país. 0 crescimento econômico em um país pobre vem acompanhado por emissões em níveis crescentes associadas ao crescimento da atividade produtiva. Devido à condição de pobreza do país, é dada uma prioridade menor sobre a qualidade ambiental, o que implica menos controle e poucas políticas ambientais sobre os níveis de emissões (ÁVILA; DINIZ, 2015).

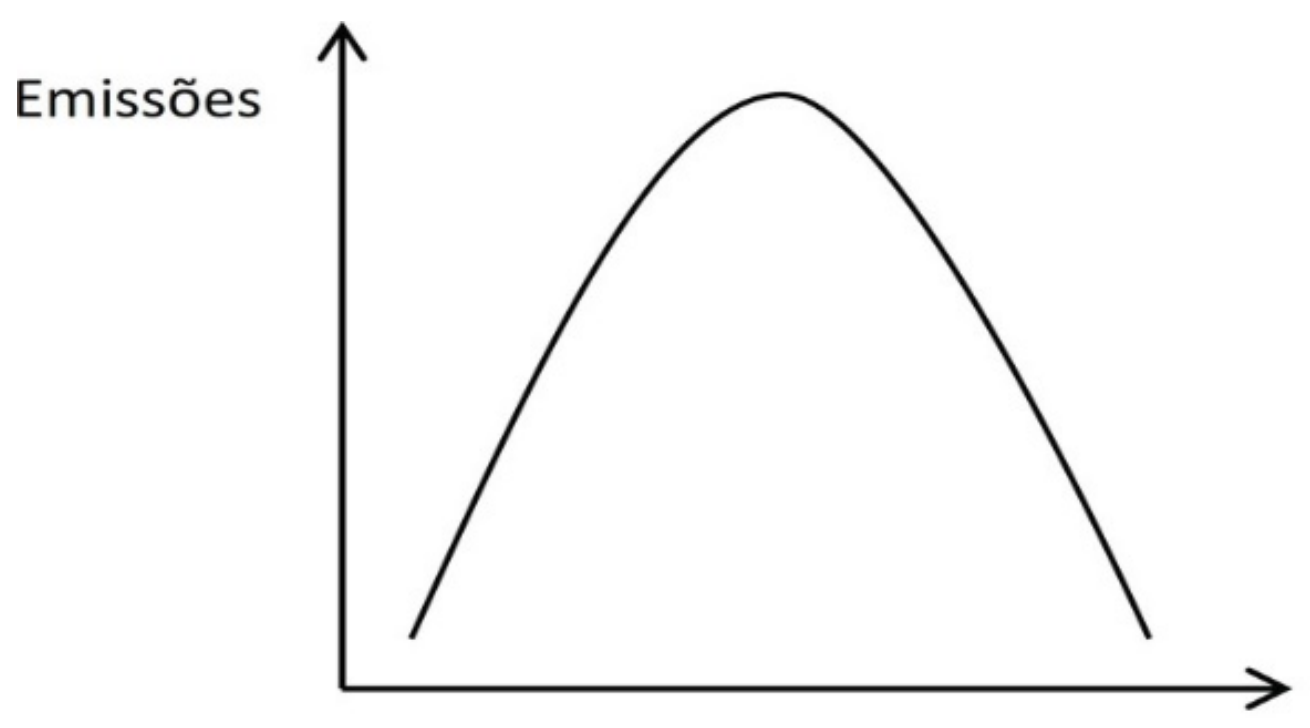

Renda

Figura 2 -Curva Ambiental de Kuznets

Fonte: Ávila; Diniz, 2015.

Segundo Romeiro (2012), Kuznets verificou se a desigualdade na distribuição de renda aumentava ou diminuía com o nível de renda do país e quais fatores determinavam o comportamento destas variáveis. Assim, o desenvolvimento não sustentável pode ser uma restrição ao crescimento da economia, mas uma restrição apenas relativa, superável indefinidamente pelo progresso científico e tecnológico.

O formato de "U-invertido", observado inicialmente por Kuznets, é explicado por dois argumentos. Arrow et al. (1995) explicam que a parte crecente da curva representa a evolução do crescimento econômico, quando os países deixaram de ser uma economia agrária "limpa" para serem uma economia industrial "poluída" e também uma economia de serviços "limpos". Ou seja, essa parte é influenciada pela atuação do mercado e pelas alterações causadas no sistema de regulação governamental. Quanto à parte decrescente da curva, Suri e Chapman (1998) explicam como sendo a forma como os países com economias em crescimento transferem seus processos produtivos com alto nível de poluição para países com economias subdesenvolvidas e permanecem com o conhecimento de serviços e de tecnologia da informação. É possível perceber que, durante todo o processo de desenvolvimento, a melhor alternativa para tentar alcançar uma qualidade ambiental para muitos países só é possível tornando-se ricos (BECKERMAN, 1992).

RELNA, Belo Horizonte - MG, Brasil, v.22, n.I, p.41-62, Jan. - Mar. 2017 - ISSN 2179-8834 


\section{Metodologia}

Para analisar a relação entre desenvolvimento sustentável e competitividade, fez-se uso de regressões lineares, visto que essa técnica permite testar as hipóteses deste trabalho. Indicadores relacionados ao tripé da sustentabilidade foram utilizados como variáveis previsoras, enquanto que o $\mathrm{GCl}$ foi usado como variável de resposta.

Também foi determinado o conjunto de regressores que formam o modelo mais adequado para previsão da competitividade através do método de seleção Stepwise. Esta técnica é amplamente utilizada para identificar quais variáveis explicativas são estatisticamente significantes para o modelo quando comparadas aos demais previsores (STEYERBERG; EIJKEMANS; HABBEMA, 1999). Os indicadores utilizados são descritos a seguir:

- IDHd (Índice de Desenvolvimento Humano ajustado para a desigualdade) Apesar de contemplar uma variável econômica (PIB per capita), este indicador leva em consideração aspectos humanos de base, como por exemplo expectativa de vida e escolaridade média, os quais estão intimamente relacionados com o nível de desenvolvimento social de um país. Além disso, o IDH tem uma abrangência muito grande, sendo publicado pela maioria dos países do globo. Portanto, este indicador foi escolhido para representar a dimensão humana neste trabalho.

- EPI (Environmental Performance Index) - O EPI é um indicador de cunho ambiental desenvolvido pela universidade de Yale, conforme discutido na seção anterior. Por conta da sua representatividade e abrangência, este indicador foi escolhido para representar aspectos relacionados ao meio ambiente nas análises.

- PIB (PPP) e PIB (US\$) (Produto Interno Bruto per capita em dólares internacionais e dólares americanos, respectivamente) - O PIB mede, em termos monetários, a quantidade de bens e serviços produzidos dentro da fronteira de um determinado país. Foram consideradas duas unidades monetárias para o PIB: dólar internacional (I\$) e dólar americano (US\$). Dólar internacional é uma moeda fictícia, que tem o mesmo poder de compra, em um determinado país, que o dólar americano nos EUA. Portanto ele leva em consideração o poder aquisitivo dos cidadãos de uma dada nação. Por meio da inclusão do PIB neste trabalho, busca-se avaliar os efeitos dos aspectos econômicos na competitividade.

- SDG (Sustainable Development Goals Index) - Conforme discutido anteriormente, este indicador contempla o desenvolvimento sustentável em todas as suas dimensões, dando uma ênfase maior aos aspectos ambientais e sociais. Ele foi incluído neste trabalho com o intuído de avaliar como se dá sua relação com a competitividade.

- GCl (Global Competitiveness Index) - O GCl foi o indicador utilizado para mensurar competitividade das nações neste trabalho, em detrimento do indicador publicado anualmente pelo IMD. O motivo para isso é que o $\mathrm{GCl}$ abrange um número muito maior de países que o indicador do IMD. 
As hipóteses testadas neste trabalho foram:

$\mathrm{H} 1$ : existe uma relação positiva entre a competitividade $(\mathrm{GCl})$ e o desenvolvimento humano (IDHd);

H2: existe uma relação positiva entre a competitividade (GCl) e desempenho ambiental (EPI);

H3: existe uma relação positiva entre a competitividade $(\mathrm{GCl})$ e o desenvolvimento econômico (PIB (US\$) e PIB (PPP));

H4: existe uma relação positiva entre a competitividade $(\mathrm{GCl})$ e o nível de sustentabilidade (SDG) de um país;

H5: a relação entre o $\mathrm{GCl}$ e o PIB (PPP) é mais significante que a relação entre o $\mathrm{GCl}$ e o PIB (US\$).

As hipóteses $\mathrm{H} 1$ a $\mathrm{H} 4$ foram testadas por meio dos coeficientes obtidos a partir de regressões simples, do teste $t$ e do valor $P$. Em termos práticos, para uma quantidade elevada de graus de liberdade, pode-se descartar a hipótese nula (inexistência de regressão entre a variável de resposta e a variável de previsora) para um teste t maior que 2 . Já o valor $P$ indica a probabilidade de se obter um valor para o teste $t$ igual ou mais extremo que o obtido, caso a hipótese nula seja verdadeira. Um valor $\mathrm{P}$ inferior a $5 \%$ é geralmente tido como satisfatório (MARTINS, 2008). Todas as análises foram feitas no software gratuito $R$. Os dados foram coletados dos sites do World Economic Forum, do World Data Bank, do United Nations Development Programme e do Yale Center for Evironmental Law and Office. 116 países foram considerados neste trabalho. Para evitar problemas durante as regressões múltiplas, os dados utilizados foram normalizados para que ficassem numa faixa de variação de $0-1$.

Os países foram então divididos em grupos por meio de uma análise de cluster. Análise de Cluster (Cluster Analysis) é uma técnica estatística multivariada, que permite ao pesquisador separar ou classificar objetos observados em um grupo ou em número específico de subgrupos ou conglomerados mutuamente exclusivos, de modo que os subgrupos formados tenham características de grande similaridade interna e grande dissimilaridade externa (MANLY, 2008).

Existem duas técnicas de cluster utilizadas, o chamado método hierárquico, segundo Tumminello, Lillo, e Mantegna (2010), em que são testadas todas as possibilidades de agrupamento, e como solução o pesquisador pode avaliar qual o a quantidade de grupos mais pertinente para sua pesquisa; e o método chamado não hierárquico, também conhecido como K-Means, conforme explica Hennig (2008), no qual o pesquisador define a priori quantos grupos deseja e o algoritmo trabalha para distribuir as observações pela quantidade de grupos pré-determinada. No presente trabalho, optou-se por utilizar o método hierárquico.

De acordo com Hair et al. (2010), uma vez definido o problema e as variáveis a serem utilizadas, o pesquisador deve seguir alguns passos para a análise de cluster hierárquica, a saber: 1) Definir uma medida de similaridade; 2) Definir qual o método de agrupamento; e 3) Definir quantos grupos farão parte da solução final.

Visando avaliar a relação entre os aspectos de competitividade e sociais/ambientais nos diferentes grupos gerados pela análise de cluster, fez-se uma análise fatorial. Esta técnica é utilizada para reduzir o número de variáveis, transformando-as em um 
número menor de fatores que podem ser mais facilmente interpretados com pouca perda de variabilidade (HAIR et al., 2010). A técnica gera vários fatores, e cabe ao pesquisador definir quantos fatores farão parte da solução final; selecionam-se os fatores que mais explicam a variância total dos dados. Em estudos acadêmicos em geral, faz-se o corte onde o autovalor (indicador de variância explicada pelo fator) é maior do que 1 (HAIR et al., 1998).

\section{Resultados}

O principal objetivo deste estudo é verificar como se dá a relação entre desenvolvimento sustentável e competitividade. Para isso, primeiramente avaliou-se o impacto dos indicadores estudados na competitividade dos países por meio de regressões simples. Em seguida, utilizou-se o método de regressão Stepwise para selecionar o conjunto de indicadores que formam o melhor modelo de previsão da competitividade. Os países foram então divididos em grupos por meio de uma análise de cluster hierárquica. Por fim, fez-se uma análise fatorial e os países foram dispostos por grupos em um gráfico de dispersão, o qual contém nos eixos os dois fatores mais representativos obtidos na análise.

\subsection{Regressão linear simples e multivariada}

A TAB 1 mostra os resultados obtidos pelas regressões simples.

Tabela 1-Resultados das regressões lineares simples: $\mathrm{GCl}=a x+b$

\begin{tabular}{lcccc}
\hline & A & teste $\mathbf{t}>\mathbf{2}$ & Valor $\mathbf{p}<\mathbf{0 . 0 5}$ & $\mathbf{r}^{2}$ ajustado \\
\hline EPI & 0,532 & 12,219 & $<2 \mathrm{E}-16$ & 0,567 \\
IDHd & 0,434 & 15,780 & $<2 \mathrm{E}-16$ & 0,683 \\
PIB (US\$) & 0,479 & 14,820 & $<2 \mathrm{E}-16$ & 0,655 \\
PIB (PPP) & 0,421 & 19,920 & $<2 \mathrm{E}-16$ & 0,775 \\
SDG & 0,590 & 16,780 & $<2 \mathrm{E}-16$ & 0,709 \\
\hline
\end{tabular}

Fonte: dados da pesquisa

Os valores de "a", na TAB 1, mostram que todos os indicadores analisados impactaram positivamente na competitividade, sendo que o SDG teve maior influência (um aumento de 100\% no SDG levou a um aumento de $59 \%$ no $\mathrm{GCl}$ em média. Esta tendência está de acordo com o que foi discutido por Herciu e Ogrean (2014), que dizem que a competitividade é um conceito que abarca a performance econômica, social e ambiental de um país. Os valores dispostos na coluna "teste t" permitem a rejeição da hipótese nula a um risco de $5 \%$, ou seja, constata-se que, de fato, há regressão entre $\circ \mathrm{GCl}$ e as variáveis analisadas (CECILIA-NICOLETA; ALEXANDRU, 2015). O valor $P$ corrobora este resultado. Tendências similares 
foram obtidas por Kasimovskaya e Didenko (2013), tendo os indicadores EPI e PIB per capita como variáveis explicativas e o $\mathrm{GCl}$ como variável de resposta.

Analisando os valores de $r^{2}$ na TAB 1, pode-se perceber que a variável com maior poder de previsão da competitividade nos países foi o PIB (PPP), o que ressalta a forte relação existente entre o poder aquisitivo dos cidadãos de uma nação e sua competitividade. Há uma diferença considerável no $r^{2}$ obtido pelo PIB (US\$) e o PIB (PPP) na explicação do $\mathrm{GCl}$, indicando que a competitividade é um conceito que vai além do poder econômico de um país, apesar de estar fortemente ligada a este, sendo também influenciada pela qualidade de vida dos seus habitantes. Num estudo feito por Balkyte e Tvaronavičiene (2010), os autores ressaltam que o principal objetivo da competitividade é de fato melhorar a qualidade de vida dos cidadãos como um todo, não se resumindo apenas a condições materiais.

O IDHd é um indicador mais abrangente que o PIB (US\$), já que ele leva em consideração o PIB per capita e outros fatores relacionados ao desenvolvimento em seu cálculo. Portanto, é natural que o IDHd tenha um poder explicativo maior sobre o GCl que o PIB (US\$). (2011) aponta que uma nação com um alto grau de competitividade é capaz de gerar um ambiente propício para a melhora da qualidade de vida dos cidadãos, o que ressalta $\mathrm{Na}$ relação existente entre $0 \mathrm{GCl}$ e o IDHd. Estes resultados indicam que as hipóteses de H1 a H5 são verdadeiras.

A TAB 2 mostra os resultados obtidos pelo método Stepwise.

Tabela 2 - Modelo Obtido pelo método Stepwise

\begin{tabular}{lcccc}
\hline & Coeficientes & Teste $\mathbf{t}$ & Valor $\mathbf{P}$ & $\mathbf{r}^{2}$ ajustado \\
\hline PIB (PPP) & 0,281 & 7,718 & $5,11 \mathrm{E}-12$ & 0,808 \\
SDG & 0,242 & 4,545 & $1,38 \mathrm{E}-05$ & --- \\
\hline
\end{tabular}

Fonte: dados da pesquisa

Os resultados sugerem que o $\mathrm{GCl}$ é melhor explicado pelos indicadores PIB (PPP) e SDG. Como já foi discutido anteriormente, o SDG é o indicador mais abrangente dentre os analisados uma vez que seus objetivos abarcam todas as dimensões do tripé da sustentabilidade. Porém, dentre os critérios considerados por esse indicador, 7 são de cunho ambiental, 7 são de cunho social/humano e apenas 2 são de cunho econômico. Portanto, o PIB (PPP) preenche a deficiência econômica do SDG. Porém, é importante ressaltar que o ganho no poder de explicação do modelo apresentado na TAB 2 em relação ao modelo de regressão simples utilizando somente o PIB (PPP) foi menor que $5 \%$, sugerindo que a dimensão econômica é a que possui relação mais forte com a competitividade dos países. Também foi constatado a partir de testes de hipótese que os resíduos possuem distribuição normal, obedecem ao princípio da homocedasticidade e não apresentam autocorrelação. Por base na distância de Cook, não foram detectados outliers de regressão. O gráfico de dispersão dos resíduos mostrou que de fato há uma relação linear do $\mathrm{GCl}$ com o SDG e o PIB (PP).

\subsection{Análise de cluster}

RELNA, Belo Horizonte - MG, Brasil, v.22, n.1, p.41-62, Jan. - Mar. 2017 - ISSN 2179-8834 
Optou-se por utilizar a metodologia hierárquica para definição dos clusters. Foi selecionada a distância euclidiana como medida de similaridade e o método de encadeamento completo como técnica de aglomeração. A padronização das variáveis, para evitar o efeito de diferentes escalas nas variáveis, foi feita pelo método Zscore.

Finalmente, foi decidido utilizar a solução de agrupamento de quatro clusters (FIG 03). Com estes resultados os clusters ficaram agrupados da seguinte maneira:

- O primeiro com 43 países: É composta por alguns países da américa do sul como Brasil, Bolívia, Peru, américa central como Costa Rica e Republica Dominicana, países da África e leste europeu. São países que se caracterizam índices medianos em EPI, IHDI, SDG e GCI.

- O segundo com 26 países: Composta por países como Itália, Espanha, Japão, Portugal, Argentina, etc. São países que se caracterizam por índices ligeiramente maiores de EPI, IHDI, SDG e GCI em relação ao primeiro grupo.

- O terceiro com 17 países: Composta por Suíça, Suécia, Noruega, Dinamarca, Estados Unidos e Reino Unido. São países que se caracterizam pelos maiores índices de EPI, IHDI, SDG e GCI.

- O último com 30 países: Composta por Bangladesh, Cambodia, Índia, Paquistão, Zimbabwe, etc. São países que se caracterizam pelos menores índices de EPI, IHDI, SDG e GCI.

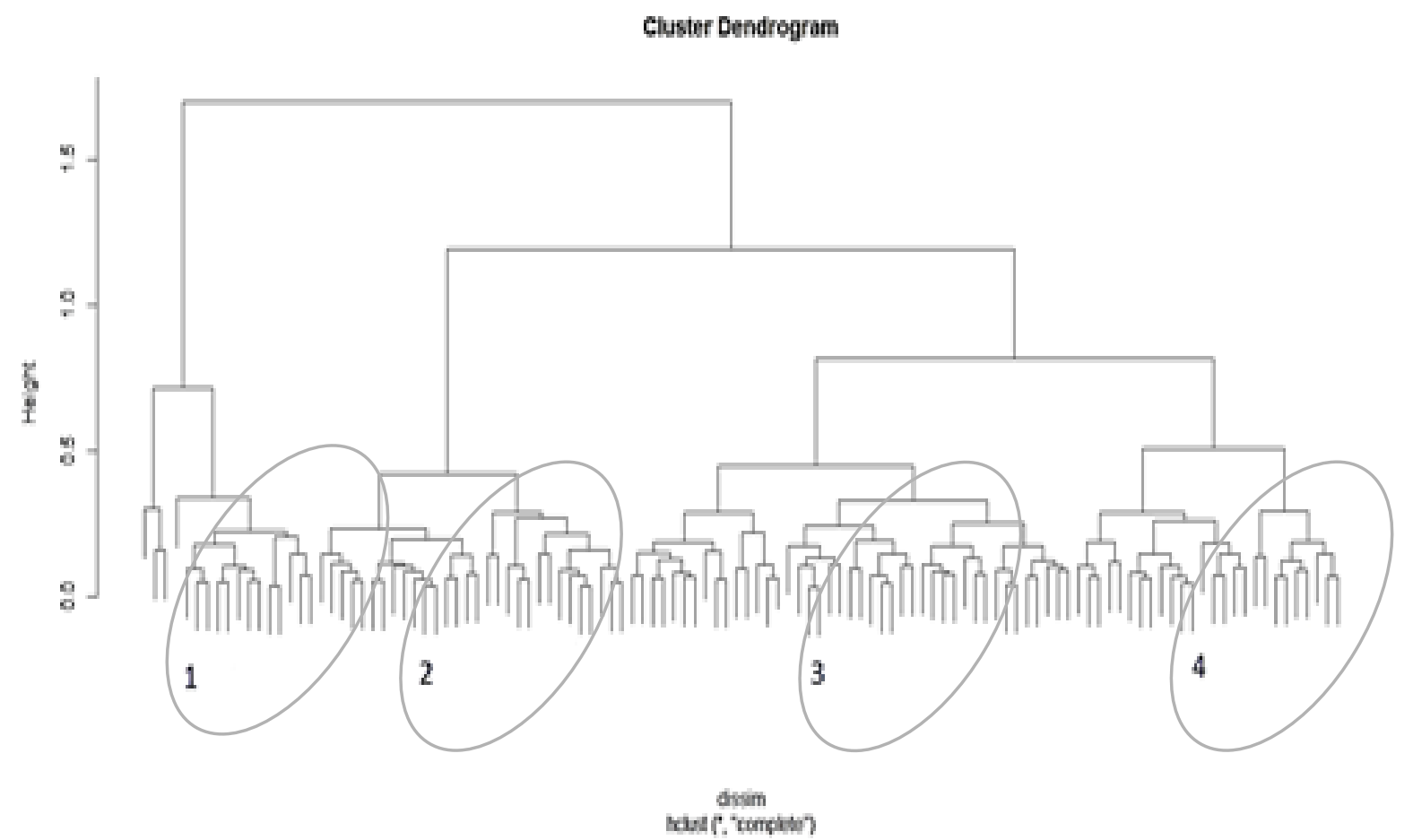

Figura 3 -Dendrograma da análise de cluster

Fonte: Dados da pesquisa, 2016. 


\subsection{Analise fatorial}

Para avaliar se os resultados da análise fatorial são adequados, inicialmente procedem-se os testes $\mathrm{KMO}$ e Bartlett.O resultado obtido pelo teste $\mathrm{KMO}$ foi 0,856 ,indicando que os dados são bem representados pela análise fatorial. Já o resultado do teste de esfericidade de Bartlett permitiu rejeitar a hipótese nula, confirmando que existe uma correlação significante entre as variáveis.

As marcações na TAB 3 abaixo indicam quais variáveis estão mais relacionadas com qual fator, com isso fica mais fácil identificar e nomear os fatores que diferenciam os países.

Tabela 3- Matriz de componentes rotacionada.

\begin{tabular}{lll}
\hline & \multicolumn{1}{l}{ Componente } & \\
\cline { 2 - 3 } EPI & $\mathbf{1}$ & $\mathbf{2}$ \\
\cline { 2 - 3 } IHDI & $\frac{913}{843}$ &, 327 \\
PIB (PPP) & $\underline{, 523}$ &, 488 \\
SDG &, 561 & $\underline{837}$ \\
PIB (U\$) & $\underline{, 316}$ &, 475 \\
GCI &, 316 & $\underline{932}$ \\
\hline
\end{tabular}

Fonte: Dados da pesquisa, 2016.

Pode-se observar que as 6 variáveis podem ser resumidas em dois fatores: Fator 1: Aspectos de desenvolvimento social e ambiental e Fator 2: Aspectos de competitividade

A FIG 04 ilustra o mapa dos países em relação ao fator 01 (desenvolvimento ambiental e social) e ao fator 02 (competitividade). 


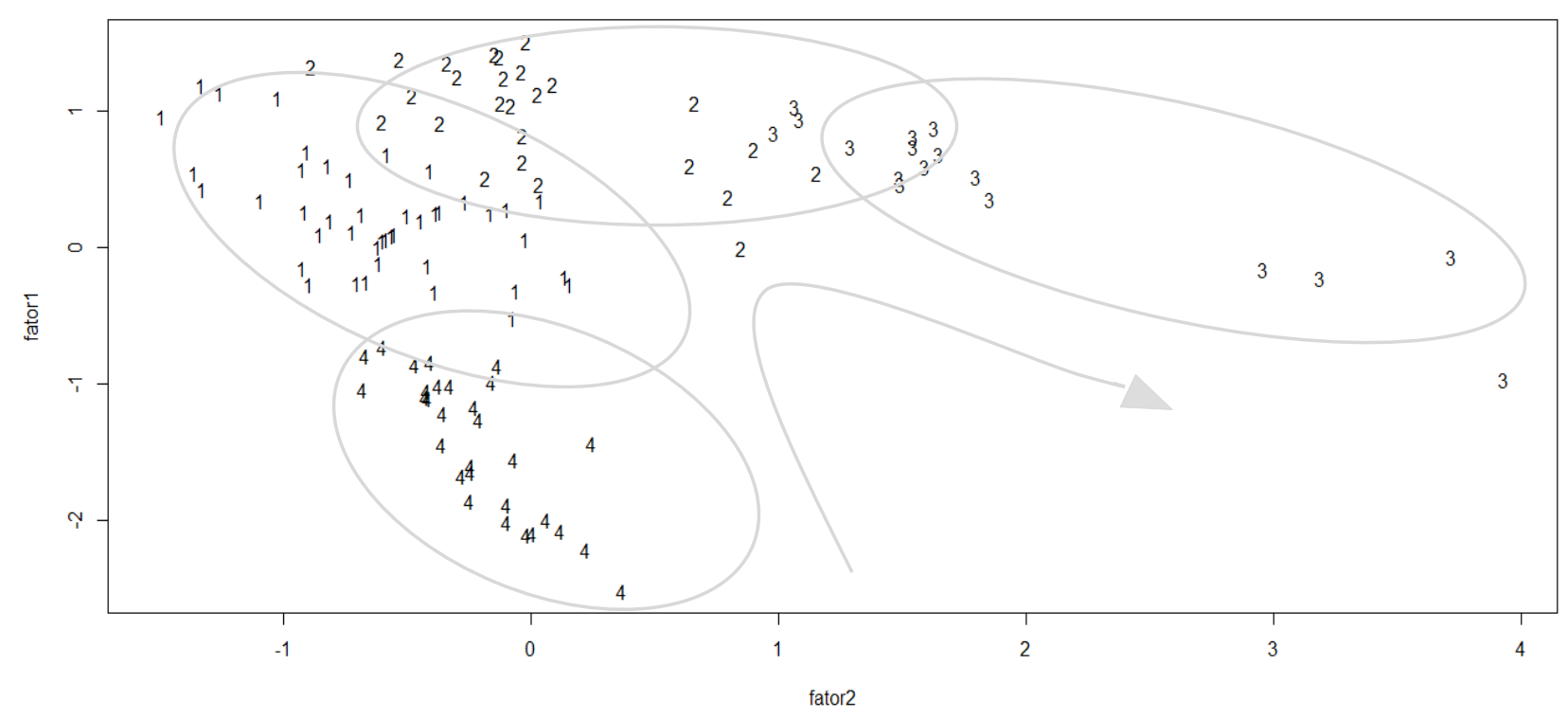

Figura 04 -Mapa dos grupos de países em relação aos aspectos ambientais/sociais (fator 01 ) e de competitividade (fator 02)

Fonte: Dados das Pesquisa, 2016.

O grupo 04 representa o grupo de países com os menores níveis de competitividade e desenvolvimento ambiental e social (dentre eles destacam-se: Etiópia, Guiana e Gana fazem parte deste grupo). No grupo 01 , constam os países que apresentam baixos níveis de competitividade e um nível mediano de desenvolvimento social e ambiental (neste grupo se situa o Brasil e a maioria dos países da América do Sul). Segundo Perkins (2001), os países em desenvolvimento são forçados a explorar seu ambiente e não podem se dar ao luxo de protegê-lo da poluição à medida que eles começam a se desenvolver. Entretanto, assim que realizam essa exploração, esses países em desenvolvimento alcançam um nível de renda em que são capazes de pagar métodos de produção ambientalmente corretos e podem aumentar os recursos governamentais dedicados à proteção do meio ambiente.

No grupo 02, encontram-se os países que apresentam um índice de desenvolvimento social e ambiental similar ou pouco melhor que os países do grupo 01, todavia com um ganho nos índices de competitividade (Espanha, Portugal, Itália e Israel fazem parte deste grupo). E, finalmente, o grupo 03 são países que apresentam índices de desenvolvimento social e ambiental similares aos dos grupos 01 e 02, entretanto, com relação aos índices de competitividade, estes países apresentam um significativo aumento (Suíça, Alemanha, Dinamarca, EUA e Canadá fazem parte deste grupo).

Nos grupos 3 e 1, existem países com níveis de desenvolvimento social e ambiental similares, entretanto, níveis de competitividade diferentes. Porter e Van Der Linde (1995) sugerem que uma regulação ambiental rigorosa sob a forma de incentivos econômicos pode desencadear inovação, que pode eventualmente aumentar a competitividade de uma empresa, podendo assim superar os custos privados em curto prazo desta regulamentação. Portanto, um caminho natural para as empresas seria na direção do grupo 3 , onde situam-se os países mais ricos detentores das organizações mais inovadoras. 
Em relação ao grupo 4, é possível visualizar que nenhum país deste grupo apresenta maiores indicadores sociais e ambientais em relação aos demais grupos. Então, pode-se dizer que nos países em estágios iniciais de desenvolvimento, a preocupação com os problemas ambientais é baixa e tecnologias amigas ao meio ambiente não estão disponíveis.

Em geral, os países dentro de cada grupo apresentam uma relação inversa entre competitividade e desenvolvimento social e ambiental. Isto leva a deduzir que o avanço da competitividade pode estar relacionado a um trade-off, onde os aspectos sociais e ambientais são afetados negativamente. Os resultados estão de acordo com os obtidos por Samimi, Ghaderi e Ahmadpour (2011), que dizem que o custo da regulação ambiental retarda o crescimento da produtividade $\mathrm{e}$ impede a competitividade nos mercados internacionais.

Por fim, pode-se fazer uma comparação entre o mapa dos grupos de países em relação aos aspectos ambientais/sociais (fator 01 ) e de competitividade (fator 02), apresentado na FIG 04, e a curva de Kuznets (seta). A curva ambiental de Kuznets postula uma relação entre emissões e renda no formato de $U$ invertido. Ou seja, em patamares iniciais do crescimento econômico, renda e emissões guardam uma relação direta. Porém, à medida que a renda cresce e chega a um determinado nível, as emissões tendem a apresentar trajetória descendente. Isso ocorre porque, em níveis maiores de renda, as pessoas estão dispostas a buscar crescimento associado a um meio ambiente menos degradado. Então, países no grupo 04 são os que possuem menores índices de competitividade e menores indicadores ambientais e sociais e, ao longo do seu crescimento econômico espera-se chegar aos países do grupo 03 com os indicadores de desenvolvimento social, ambientais satisfatórios e constantes e melhores índices de competitividade.

\section{Conclusões}

As análises mostram que os indicadores avaliados impactam positivamente na competitividade. Tal tendência é conhecida como hipótese do ganha-ganha, a qual afirma que uma vantagem competitiva pode sim vir acompanhada de uma boa performance ambiental (SOUZA, 2002). O coeficiente de regressão positivo entre os indicadores EPI (desempenho ambiental) e $\mathrm{GCl}$ (índice de competitividade) ilustra essa tendência.

Dentre as dimensões do tripé da sustentabilidade, a dimensão ambiental foi a que mostrou uma relação mais fraca com a competitividade, enquanto que a dimensão econômica foi a que demonstrou um maior poder explicativo sobre o $\mathrm{GCl}$. Tendências similares foram encontradas por Kasimovskay e Didenko (2013). Isso pode ser explicado pelo fato de os pilares considerados no cálculo do $\mathrm{GCl}$ terem uma relação muito maior com fatores econômicos que com fatores ambientais ou sociais.

Ainda que não tenha sido o indicador com maior poder explicativo sobre o $\mathrm{GCl}$, o SDG mostrou um $r^{2}$ considerável (aproximadamente 0,71 ) e o maior impacto sobre a competitividade dentre os indicadores analisados. Portanto, pode-se concluir que de fato existe uma relação significante entre desenvolvimento sustentável e competitividade. A existência de tal relação é defendida por Balkyte e 
Tvaronavičiene (2010), Herciu e Ogrean (2014), Kasimovskaya e Didenko (2013) e Lapinskiené (2011).

Ao analisar o comportamento dos dados por grupos, verifica-se a existência de um trade-off entre os aspectos sociais/ambientais e de competitividade. Constatou-se ainda que há uma tendência de evolução dos países se deslocando do grupo 4 (países subdesenvolvidos como Etiópia, Guiana e Gana) ao grupo 3 (países desenvolvidos como Suécia, Noruega, Dinamarca, Estados Unidos). Esses resultados estão de acordo com o que foi discutido por Samimi, Ghaderi e Ahmadpour (2011) e com a curva de Kuznets: o crescimento econômico, após um certo nível, tem relação inversa com os níveis de emissões (Arrow et al.,1995).

Para trabalhos futuros, recomenda-se a inclusão de indicadores relacionados aos pilares de sofisticação e inovação para suprir a deficiência de previsão no modelo obtido pelo método Stepwise. Sugere-se também a replicação deste estudo longitudinalmente para minimizar eventuais impactos pontuais nos países analisados.

\section{Referências}

ARROW, K.; BOLIN, B.; COSTANZA, R.; DASGUPTA, P.; FOLKE, C.; HOLLING, C. S.; JANSSON, B. O.; LEVIN, S.; MALER, K. G.; PERRINGS, C.; PIMENTEL, D. Economic growth, carrying capacity and the environment. Science, v. 268, n. 5217, p.520-521.1995.

ARSLAN, N.; TATHDIL, $H$. Defining and measuring competitiveness: a comparative analysis of Turkey with 11 potential rivals. International Journal of Basic \& Applied Sciences, v.12, n.2, p. 31-43, 2012.

ÁVILA, E. S.; DINIZ, E. M. Evidências sobre curva ambiental de Kuznets e convergência das emissões, Estud. Econ, (São Paulo), v. 45, n. 1, jan-mar, 2015.

BALKYTE, A.; TVARONAVIČIENE, M. Perception of competitiveness in the context of sustainable development: Facets of "sustainable competitiveness". Journal of Business Economics and Management, v. 11, n. 2, p. 341-365, 2010.

BECKERMAN, W. Economic Growth and the Environment: Whose Growth? Whose Environment. World Development, v. 20, p. 481-496, 1992.

BM\&FBOVESPA. Pacto Global da ONU reconhece a diretora de Imprensa e Sustentabilidade da BM\&FBOVESPA como uma "2016 Local SDG Pioneer". São Paulo, Jun/ 2016.

CECILIA-NICOLETA, J.; ALEXANDRU, B. Perception Of Global Competitiveness In The Context Of Sustainable Development: The Cases Of Romania And Bulgaria. Anais do "ConstantinBrâncuşi". Universidade de TârguJiu, Série Economia, v.1, n. 1, 2015.

DOCUMENTOS DAS NAÇÕES UNIDAS. 1987. Nosso Futuro em Comum, capítulo 2: Rumo ao Desenvolvimento Sustentável. Disponível em: <http://www.undocuments.net/ocf-02.htm> Acesso em: 02 out. 2016. 
EDMONDS, T. Regional Competitiveness \& the Role of the Knowledge Economy, House of Commons Library, Research paper. London: Research Publications Office, p.73-55, 2000.

FERNANDES, L. A. O. The Meaning of Sustainability: Searching for Agrienvironmental Indicators. Manchester: University of Manchester - Institute for development policy and management, 2004. (Doctoral thesis).

FÓRUM ECONÔMICO MUNDIAL (2010), Disponível em: $<$ www3.weforum.org/docs/WEFGlobalCompetitivenessReport2010-11.pdf >Acesso em: 25/10/2016.

HAIR, J. F.; BLACK, W. C.; BABIN, B. J.; ANDERSON, R. E. Multivariate Data Analysis. Pearson, Ed. 2010.

HAIR, J.; ANDERSON, R.; TATHAM, R.; BLACK, W. Multivariate data analysis. 5 ed., ed. New Jersey: Prentice Hall, 1998 cap.1-3. 768p.

HENNIG. Dissolution point and isolation robustness: robustness criteria for general cluster analysis methods. Journal of Multivariate Analysis, v.99, p.1154-1176, 2008.

HERCIU, M.; OGREAN, C. An Overview on European Union Sustainable Competitiveness. Procedia Economics and Finance, v. 16, p. 651-656, maio, 2014.

HSU et al. Global Metrics for the Environment. Disponível em: <http://epi.yale.edu/downloads>. Acesso em 05 out. 2016.

IMD. International Institute for Management Development. World competitiveness yearbook 2016, Disponível em: <https://goo.gl/ypJ6b5> Acesso em: 04 de out. 2016.

KASIMOVSKAYA, E.; DIDENKO, M. International Competitiveness and Sustainable Development: are they apart, are they together? A quantitative approach. v. 2, p. 37-51, 2013.

KEMERICH, P. D. C.; RITTER, L. G.; BORBA, W. F. Indicadores de sustentabilidade ambiental: métodos e aplicações. Revista Monografias Ambientais - REMOA, v. 13, n. 5 (2014): Edição Especial LPMA/UFSM, p. 3723-3736, Santa Maria, 2014.

LAPINSKIENÉ, G.. The analysis of the relationship between the sustainable development and competitiveness in the European countries. Intellectual Economics. v. 5, n. 3, p. $434-444,2011$

MALHEIROS, T. F.; PHILIPPI JR., A.; COUTINHO, S. M. V. Agenda 21 nacional e indicadores de desenvolvimento sustentável: contexto brasileiro. Revista Saúde e Sociedade, São Paulo, v. 17, n. 1, p.7-20, mar, 2008.

MANLY B. J. F. Métodos estatísticos multivariados: Uma introdução. Porto Alegre: Artmed. 2008.

MARCONDES, A. W.; BACARDJI, C. D. ISE - Sustentabilidade no mercado de capitais, 1 Edição, Report Editora, São Paulo, 2010.

MARTINS, G. A. Estatística Geral e Aplicada. 3. ed. São Paulo: Atlas., 2008.

ONU. Divisão para o Desenvolvimento Sustentável. Indicators of sustainable development: guidelines and methodologies: 2001. Disponível em: <http://www.un.org/esa/sustdev/isd.htm>. Acesso em: 05 out. 2016. 
PEDRINI, A. G. Educação Ambiental: reflexões e práticas contemporâneas. Rio de Janeiro: Vozes, 1998.

PERKINS, D. H. Economics of Development, 5th Edition. New York: W.W. Norton \& Company, Inc. 2001.

PHILIPPI, L. S. A Construção do Desenvolvimento Sustentável. In.: LEITE, A. L. T. A.; MININNI-MEDINA, N. Educação Ambiental (Curso básico a distância), Questões Ambientais - Conceitos, História, Problemas e Alternativa. 2. ed, v. 5. Brasília: Ministério do Meio Ambiente, 2001.

PORTER, M. C.; VAN DER LINDE, "Toward a new conception of the environment competitiveness relationship", Journal of Economic Perspectives, v.9, p.97-118, 1995.

PORTER, M. E. Competition in Global Industries. Harvard, Harvard University Press. 1986.

PROGRAMA DAS NAÇÕES UNIDAS PARA O DESENVOLVIMENTO (PNUD). Declaração do Milênio. 2000. Disponível em:< https://goo.gl/Sq84NX > . Acesso em: 09 out. 2016.

ROMEIRO, A. R. Desenvolvimento Sustentável: uma perspectiva econômicoecológica. Estud (São Paulo), v. 26, n. 74, 2012. Disponível em: <http://dx.doi.org/10.1590/S0103-40142012000100006> Acesso em: 10 de nov. 2016

SALA-I-MARTIN, X.; BILBAO; OSORIO, B.; BLANKE, J.; HANOUZ, M.; GEIGER, T.; KO, C. The Global Competitiveness Index 2013-2014: Sustaining Growth, Building Resilience, The Global Competitiveness Report 2013-2014. World Economic Forum, 2013, 3-551;

SALA-I-MARTIN, Xavier et al. The global competitiveness index 2009-2010: Contributing to long-term prosperity amid the global economic crisis. The global competitiveness report, v. 2010, p. 3-47, 2009.

SAMIMI, A. J.; GHADERI, S.; AHMADPOUR, M. Original article. Environmental Sustainability and Economic Growth: Evidence from Some Developing. v. 5, n. 5, p. 961-966, 2011.

SANCHS, I. Estratégias de Transição para do século XXI - Desenvolvimento e Meio Ambiente. São Paulo: Studio Nobel - Fundação para o desenvolvimento administrativo, 1993.

SCHLINDWEIN, M. M.; JUCHEM, D. M.; RAFUL, N. Desempenho ambiental como fator de competitividade das empresas goianas. In: Congresso Da Sociedade Brasileira De Economia, Administração E Sociologia Rural, 47., 2009, Porto Alegre. Anais... Porto Alegre: SOBER, 2009. CD-ROM.

SOUZA, C. F.; BRITO, M. A. Implicações socioambientais da geração de resíduos sólidos pelas microempresas de transformação em Juazeiro do Norte - CE. Revista Economia em Debate, Crato, n. 2, p. 148-170, 2008.

SOUZA, R. S. Evolução E Condicionantes Da Gestão Ambiental Nas Empresas. REAd - Revista Eletrônica de Administração, v. 8, n. 6, p. 1-22, 2002.

STEYERBERG, E. W.; EIJKEMANS, M. J. C.; HABBEMA, J. D. F. Stepwise 
Selection in Small Data Sets: A Simulation Study of Bias in Logistic Regression Analysis. Journal of Clinical Epidemiology, v. 52, n. 10, p. 935-942, 1999.

SURI, V.; CHAPMAN, D. Economic growth, trade and the energy: Implications for the environmental Kuznets curve. Ecological Economics, New York, v. 25, p. 195-208. 1998.

TUMMINELLO, M.; LILLO, F.; MANTEGNA, R. N. Correlation, hierarchies, and networks in financial markets. Journal of Economic Behavior \& Organization, v.75, n.1, p.40-58, 2010.

UNITED NATIONS DEVELOPMENT PROGRAMME. Human Development Reports. Disponível em: <http://hdr.undp.org/>. Acesso em: 05 out. 2016.

UNITED NATIONS, Sustainable Development Goals. Disponível em:<https://sustainabledevelopment.un.org/sdgs>. Acesso em: 05 de outubro de 2016.

VIEIRA, T. M. P.; LIMA, G.B.A.; BARROS, S.R.S. Proposta de indicadores de sustentabilidade para o setor de distribuição de combustíveis: o caso da Petrobras Distribuidora. In: XXVIII ENCONTRO NACIONAL DE ENGENHARIA DE PRODUÇÃO, 28, 2008, Rio de Janeiro, Anais... Rio de janeiro, , 2008, p. 1-17. <http://www.abepro.org.br/biblioteca/enegep2008_TN_STO_079_550_11291.pdf> Acesso em: 05 de outubro de 2016 